\title{
Bentall Surgery for Aortic Root Aneurysm in a Patient with Marfan Syndrome
}

\author{
Mohammad Fazle Maruf ${ }^{1}$, Nazmul Hossain $^{2}$, Tahmina Akter $^{1}$, Asif Ahsan Chowdhury ${ }^{1}$, Kamrul $^{2}$ \\ hasan', Rezaul karim ${ }^{2}$,NAKamrul Ahsan ${ }^{2}$ \\ ${ }^{1}$ Department of Cardiothoracic Surgery, Chittagong Medical College, \\ ${ }^{2}$ Department of Cardiac surgery, NICVD
}

\author{
Key Words : \\ Marfan \\ Syndrome, Aortic \\ aneurysm, \\ Bentall's \\ operation, Aortic \\ valve.
}

\begin{abstract}
:
A 34 yrs old man of marfan syndrome, visited us with the complaints of palpitation, easy fatigability and chest discomfort for last 1year. He was pointed out to have the aortic root enlargement with severe aortic regurgitation \& mild mitral regurgitation by echocardiography and CT angiogram. We excised the aneurysm, implanted a composite graft with prosthetic valve conduit (Bentall operation), directly attached the coronary arteries to the aortic graft, and made the distal anastomosis to the divided aorta. Postoperative course was uneventful. To our knowledge, this is the first successful case of Bentall operation in national Institute of cardiovascular Diseases.
\end{abstract}

(Cardiovasc. j. 2015; 8(1): 78-81)

\section{Introduction}

Marfan syndrome is an autosomal-dominant hereditary connective tissue disorder with the clinical manifestations involving the ocular, skeletal, and cardiovascular systems. ${ }^{1}$ When Marfan published the first report of this condition in 1896, he emphasized primarily the ocular and skeletal abnormalities. ${ }^{2}$ The cardiovascular aspects of Marfan syndrome were first described by McKusick in $1955 .^{3}$ The cardiovascular manifestations include aortic root dilatation, aortic valvular insufficiency, mitral valve prolapse, mitral regurgitation, aortic dissection, and aortic rupture. Rupture of an ascending aortic aneurysm is the most common cause of death in patients with Marfan syndrome, while type-A aortic dissection is the second most common fatal lesion. ${ }^{4}$ We report our results of the surgical management of the aortic aneurysm with severe aortic regurgitation \& mild mitral regurgitation.

\section{Case report:}

A man of 37 yrs came to us from Munshigonj with symptoms of palpitation, dyspnea, and chest pain for one year. In addition to his positive family history of Marfan syndrome, he had the clinical findings of long slender body stature, arachnodactyly and high arched palate. A systolic murmur was heard in the mitral area and an early diastolic murmur was detected at the left sternal border. His blood pressure was 120/20 mm of $\mathrm{Hg}$. Chest radiography showed cardiomegaly and a dilated ascending aorta. Trans-esophageal echocardiography showed severe aortic regurgitation and mild mitral regurgitation. CT Angiogram revealed that the aortic root was dilated (length $84 \mathrm{~mm} \times$ transeverse $74 \mathrm{~mm} \times$ anteroposterior $70 \mathrm{~mm}$ ) sparing aortic arch and descending aorta. No evidence of aortic dissection was present. Coronary arteries were normal. His left ventricular function was good, with an ejection fraction of $60 \%$. He was in New York Heart Association functional class II.

Elective surgery was performed under Cardiopulmonary bypass (CPB). During surgery Cardio Pulmonary bypass was established through ascending aortic (distal to the aneurysm) and two stage single venous cannulation in right atrium. The ascending aorta was separated from the main pulmonary artery. Moderate hypothermia achieved $\&$ aortic cross clamp applied. The ascending aorta was opened longitudinally and selective antegrade cardioplegia was given to arrest the heart, no false lumen or thrombosis was found. The aortic annulus was dilated. The mitral valve was tested through the aorta and coaptation of the leaflets was found to be good. Aortic valve was excised and aorta was

Address of Correspondence: Dr. Mohammad Fazle Maruf, Department of Cardiothoracic Surgery, Chittagong Medical College.Chittagong, Bangladesh. Email-mfmaruf@yahoo.com 
resected. After resection of the aortic valve and the ascending aorta, the technique of Bentall procedure (Composite graft replacement of the ascending aorta with aortic valve replacement and re-implantation of the coronary arteries into the graft) was performed with $25 \mathrm{~mm}$ ATS composite bi-leaflet metallic Aortic valve conduit. Coronary orifice holes were made in the tube graft with a wire electrocautery. The coronary ostia were anastomosed to the graft using $6 / 0$ polypropylene running sutures. The aortic arch (proximal to brachiocephalic trunk) was found to be intact and it was anastomosed to the distal end of the graft. In addition, composite graft was wrapped with native aortic wall. Cross clamp time was 108 mins. Re-exploration was done on the same day for postoperative bleeding ( $2900 \mathrm{ml}$ in 5 hours). On re-exploration generalized oozing was found and haemostasis was secured. Rest of the post operative period was uneventful \& he was discharged on $11^{\text {th }}$ post operative day. The patient made a good recovery and has been followed up for 2 years.

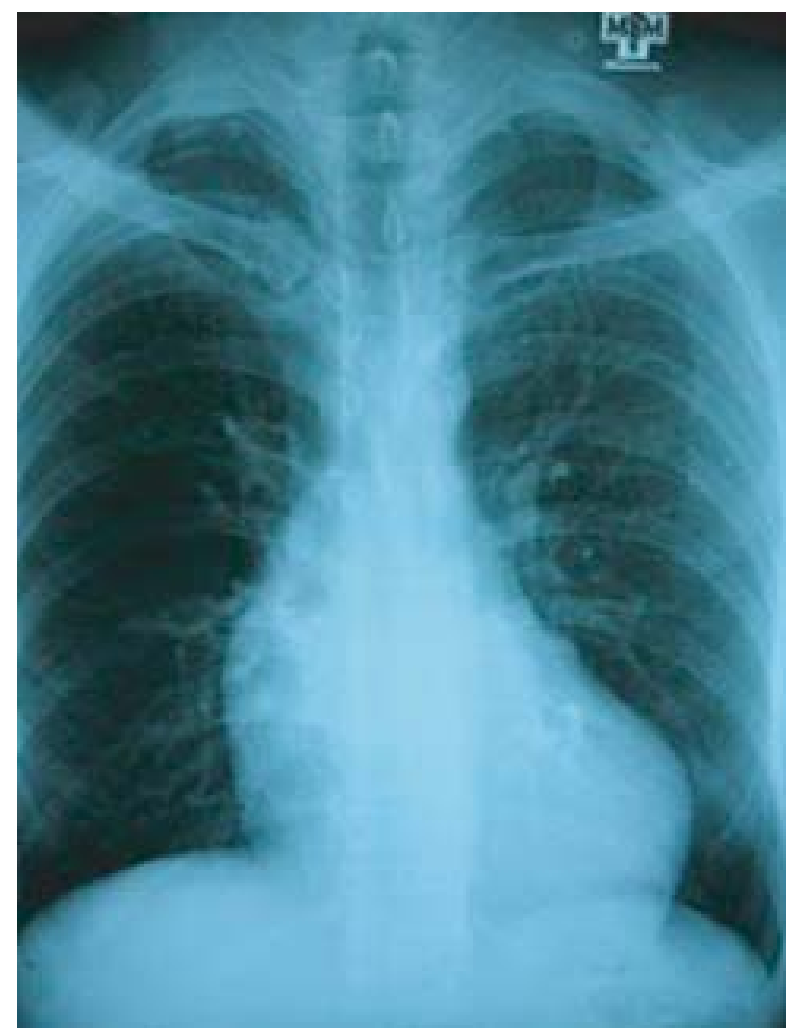

Fig.-1. $X$-ray of chest $P$-A view showed dilatation of aortic root.

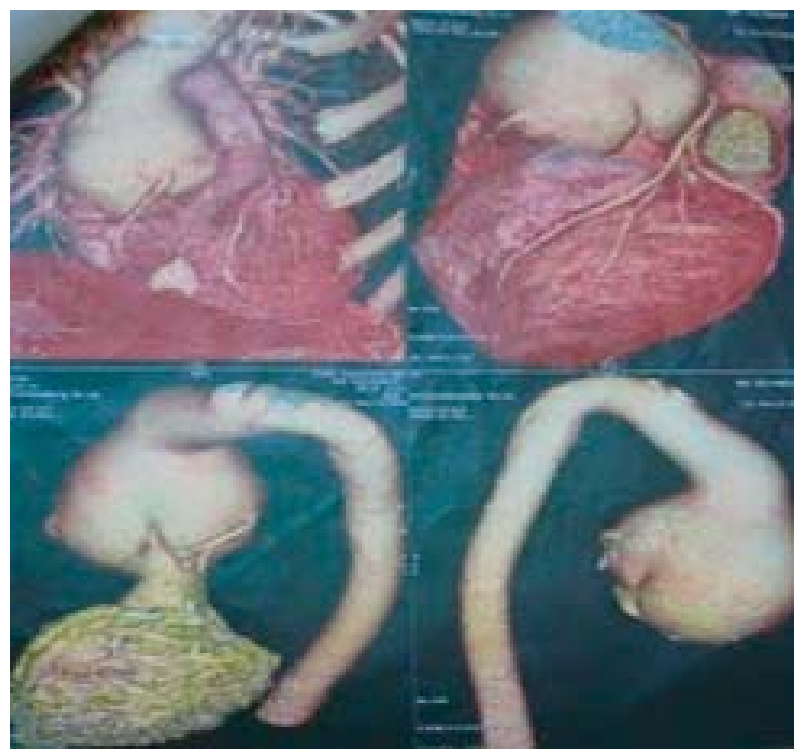

Fig.-2: CT angiogram of heart showing aneurysmal dilatation of ascending aorta.

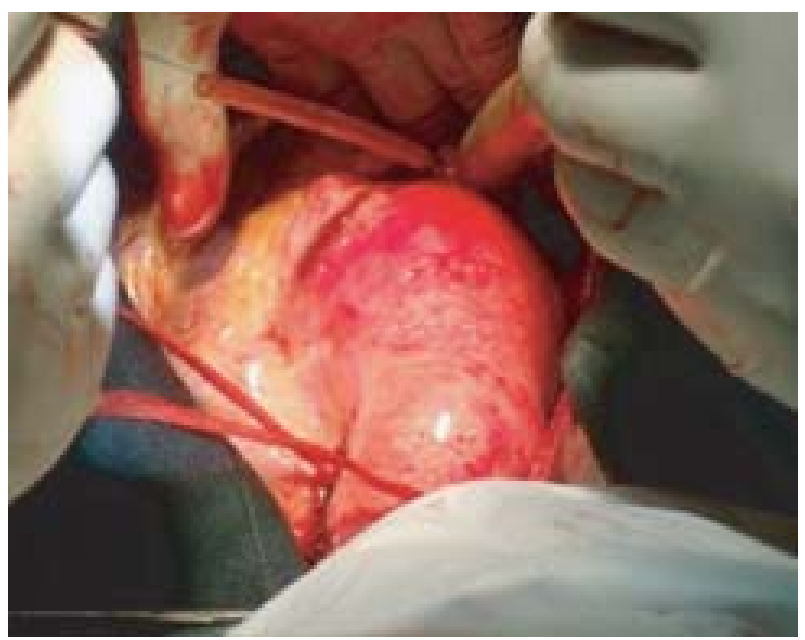

Fig.-3: Photograph of aortic aneurysm.

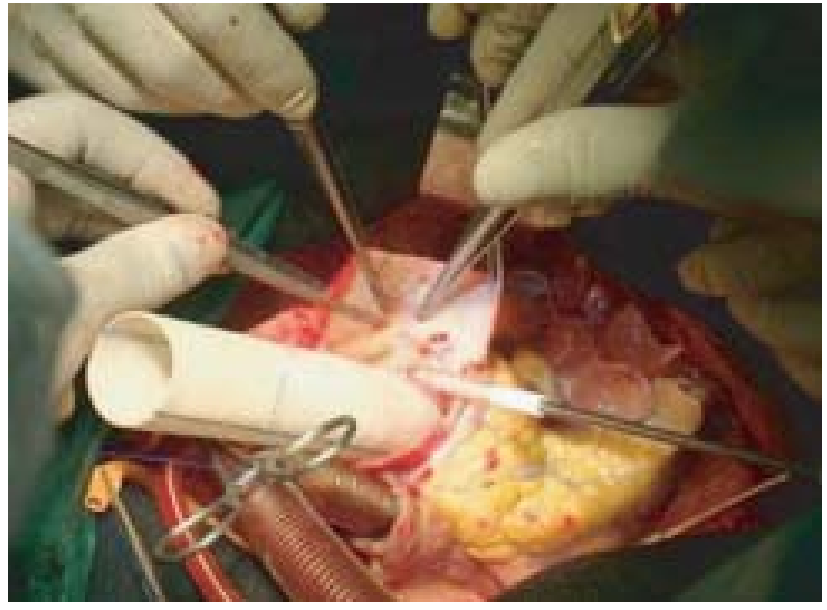

Fig.-4: Composite aortic graft with aortic valve also showing right coronary ostium. 


\section{Discussion:}

Marfan syndrome occurs with a frequency of $1 /$ 10,000 in all populations and it is often undiagnosed during life. The prevalence of a positive family history has been reported as $65 \%$ to $80 \% .{ }^{5}$ Current criteria for diagnosis depend on manifestations in the cardinal organ systems (the eye, the skeleton, the heart, and the aorta) as well as the family history. The most common cardiovascular features are mitral valve prolapse and dilation of the sinuses of Valsalva. ${ }^{5}$ The cardiovascular sequel of fibrillin deficiency appear to relate directly to weakening of the supportive tissues. ${ }^{6}$ Mitral valve prolapse and mitral valve annular dilation are age dependent and more common in women and children with Marfan syndrome. ${ }^{7}$ Aortic disease is a serious cardiovascular manifestation in individuals with Marfan syndrome and potentially fatal complication. Aortic root dilation may be the initial aortic abnormality, consisting of dilation of the sinuses of Valsalva as well as expansion of the sinotubular junction and the aortic annulus. The histopathology of the aortic wall is characterized by widespread fragmentation of the elastin component and the elastin fibers are often thin. Aortic dissection, which is common in Marfan syndrome, is usually due to an intimal tear in the proximal ascending aorta with the dissection involving the sino-tubular junction and aortic sinuses, resulting in prolapse of one or more commissures. However, the dissection never crosses or involves the annulus or cusps. Aortic valvular insufficiency results from progressive annular dilatation or acute aortic dissection. ${ }^{8}$ If such patients are not treated, associated clinical problems of aortic regurgitation and aortic dissection account for most of the early mortality and the usual age at death is in the fourth or fifth decades. ${ }^{9}$ Rupture of an ascending aortic aneurysm is the most common cause of death in older Marfan syndrome patients and type-A aortic dissection is the second most common fatal lesion. ${ }^{10}$

Management of such patients should aim to prevent symptoms, limit the risk of rupture, and prevent secondary irreversible structural changes. The only guidance to be found in the literature to help identify patients most at risk is the dimension of the aortic root. This can be obtained easily and noninvasively in most patients by transthoracic echocardiography. Echocardiographic evidence of cardiovascular involvement exists in over $95 \%$ of adults with Marfan syndrome. The annual increase in aortic size is greater in patients with aortic complications or a family history of aortic dissection. The growth rate ranges from 0.1 to $0.3 \mathrm{~cm}$ per year in patients with complications and from 0 to $0.3 \mathrm{~cm}$ per year in patients without complications. A trend towards more frequent complications in patients before the age of 40 years has also been observed. ${ }^{11}$ Surgical reconstruction is the only definitive method of managing this problem. Aortic regurgitation often appears in adults with an aortic annular diameter of $50 \mathrm{~mm}$. The risk of dissection increases with the size of the aorta (above $55 \mathrm{~mm}$ ) and many surgeons have adopted the criterion of a 50 to 55 $\mathrm{mm}$ maximal aortic root dimension for performing elective composite graft repair in patients with Marfan syndrome, although patients with a family history of aortic dissection should have surgery at the lower end of this range. Pyeritz ${ }^{12}$ demonstrated that a positive family history of dissection is a strong predictor of aortic dissection.

The ideal operation for this condition should eliminate all abnormal tissue, thereby preventing or minimizing the risk of aneurysmal recurrence or rupture. We believe that the surgical procedure of choice for patients with Marfan syndrome who have an ascending aortic aneurysm, with or without dissection, should be ascending aorta replacement with the combination of aortic valve replacement and coronary ostial reimplantation as described by Bentall and De Bono in $1968 .{ }^{13}$ This approach is preferred to prevent progressive dilatation of the sinuses of Valsalva, which could manifest as aortic insufficiency in patients who undergo other procedures such as annuloplasty, aortoplasty, endto-end primary aortic anastomosis, or isolated aortic valve replacement. ${ }^{14,15}$ Aortic valvular reparative procedures appear to be unsuitable in Marfan patients because of the high likelihood of progressive annular or sinus dilatation leading to aortic valvular insufficiency. The aortic root dimension and its rate of increase are the best indications for surgery. We regard $5.5 \mathrm{~cm}$ as the upper limit of safety and we are inclined to advocate surgery at an earlier stage in high-risk patients. 


\section{Conclusion:}

This is the case report that documents the first Bentall surgery done in National Institute of Cardiovascular Diseases, Dhaka, Bangladesh as we know. We believe that the patient for Bental surgery had significantly more advanced disease. We should consider all patients of ascending aortic root aneurysm with aortic regurgitation can be treated safely here.

\section{Conflict of Interest - None.}

\section{References:}

1. Pyeritz RE, Mckusick VA. The Marfan syndrome diagnosis and management. $N$ Engl $J$ Med 1979;300:772-777.

2. Marfan A B. Un Cas de derormation congenitale des quatres members, plus prononcee aux extremites,caracterisee par l'allongement des os avec un certain degre d'amincussement. Bull Soc Chir Paris 1896;13:220-225.

3. Mckusick VA. The cardiovascular aspects of Marfan's syndrome: a heritable disorder of connective tissue. Circulation 1955;11:321-342.

4. Peltenon L. Genetic basis of Marfan syndrome. In: Hetzer R, Gehele P, Ennker J. Eds. Cardiovascular aspects of Marfan syndrome. Darmstadt: Steinkoff Verlag, 1995;12:9-15.

5. Marsalese DL, Moodiew DS, Vacante M, Lytle BW, Gill $\mathrm{CC}$, Sterba R et al. Marfan's syndrome: natural history and long term follow up of cardiovascular involvement. J Am Coll Cardiol 1989;14:422-428.
6. Lee B, Godfrey M, Vitale E, et al. Linkage of Marfan syndrome and a phenotypically related disorder to two different fibrillin genes. Nature 1911; 352:337-339.

7. Glesby MJ, Pyeritz RE. Association of mitral valve prolapse and systemic abnormalities of connective tissue. A phenotypic continuum. JAMA 1989 Jul 28; 262(4):523-528.

8. Zhu L, Vranckx R, Van Kien PK, Lalande A, Boisset N, Mathieu $\mathrm{F}$ et al. Mutations in myosin heavy chain 11 cause a syndrome associating thoracic aortic aneurysm/ aortic dissection and patent ductus arteriosus. Nat Genet 2006 Mar;38(3):343-349.

9. Smith JA, Fann JL, Miller DC, Moore KA, DeAnda A, Mitchell RS et al. Surgical management of aortic dissection in patients with the Marfan syndrome. Circulation 1994;90(Suppl II):235-242.

10. Murdoch JL, Walker BA, Halpern BL, Kuzma JW, Mckusick. Life expectancy and causes of death in Marfan syndrome. N Engl J Med 1972; 286:804-808.

11. Stout M. The Marfan syndrome: implications for atheletes and their echocardiographic assessment. Echocardiography 2009 Oct; 26(9):1075-1081.

12. Gott VL, Cameron DE, Alejo DE, Greene PS, Shake JG, Caparrelliet DJ et al. Aortic root replacement in 271 Marfan's patients: a 24 year experience. Ann Thorac Surg 2002; 73: 438-443.

13. Bental HH, De Bono A. A technique for complete replacement of the ascending aorta. Thorax 1968; 23: 338-339.

14. Treasure T. Elective replacement of the aortic root in Marfan's syndrome [editorial]. Br Heart J 1993; 69: 101-103.

15. Roman MJ, Rosen Se, Kramer-Fox R, devereux RB. Prognostic significance of the pattern of qaortic root dilatation in the Marfan syndrome. J Am Coll Cardiol 1993;22:1470-1476. 